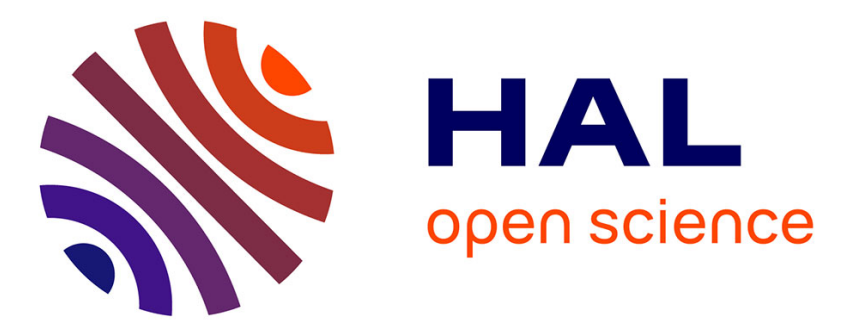

\title{
Nuclear magnetic resonance reveals "forbidden" symmetries in quasicrystals and related metallic alloys with giant unit cells
}

Peter Jeglic, Martin Klanjsek, Janez Dolinsek

\section{To cite this version:}

Peter Jeglic, Martin Klanjsek, Janez Dolinsek. Nuclear magnetic resonance reveals "forbidden" symmetries in quasicrystals and related metallic alloys with giant unit cells. Philosophical Magazine, 2007, 87 (18-21), pp.2687-2692. 10.1080/14786430701355141 . hal-00513833

\section{HAL Id: hal-00513833 \\ https://hal.science/hal-00513833}

Submitted on 1 Sep 2010

HAL is a multi-disciplinary open access archive for the deposit and dissemination of scientific research documents, whether they are published or not. The documents may come from teaching and research institutions in France or abroad, or from public or private research centers.
L'archive ouverte pluridisciplinaire HAL, est destinée au dépôt et à la diffusion de documents scientifiques de niveau recherche, publiés ou non, émanant des établissements d'enseignement et de recherche français ou étrangers, des laboratoires publics ou privés. 


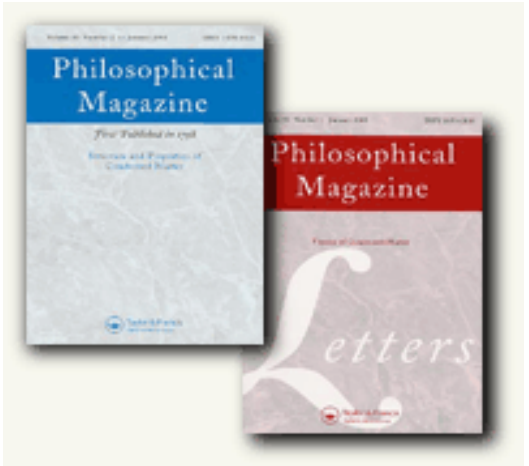

\section{Nuclear magnetic resonance reveals Â $\llbracket$ forbiddenÂ $\square$ symmetries in quasicrystals and related metallic alloys with giant unit cells}

\begin{tabular}{|c|c|}
\hline Journal: & Philosophical Magazine \& Philosophical Magazine Letters \\
\hline Manuscript ID: & TPHM-06-Aug-0348.R2 \\
\hline Journal Selection: & Philosophical Magazine \\
\hline $\begin{array}{r}\text { Date Submitted by the } \\
\text { Author: }\end{array}$ & 26-Jan-2007 \\
\hline Complete List of Authors: & $\begin{array}{l}\text { Jeglic, Peter; Jozef Stefan Institute, Solid State Physics Department } \\
\text { Klanjsek, Martin; Jozef Stefan Institute, Solid State Physics } \\
\text { Department } \\
\text { Dolinsek, Janez; Jozef Stefan Institute, Solid State Physics } \\
\text { Department }\end{array}$ \\
\hline Keywords: & magnetic resonance, quasicrystals \\
\hline Keywords (user supplied): & angular-dependent spectrum, electric field gradient, giant unit cell \\
\hline
\end{tabular}

\section{(s) ScholaroNE \\ Manuscript Central}




\begin{abstract}
We report on measuring angular dependences of the selectively excited NMR intensity in decagonal AlNiCo quasicrystal, icosahedral AlPdMn quasicrystal and orthorhombic $\xi^{\prime}$-AlPdMn single crystal with approximately 258 atoms in the unit cell. In all three cases we found 10-fold rotation patterns in agreement with structural properties of investigated samples. However, in orthorhombic $\xi$-AlPdMn complex metallic alloy a non-perfect 10-fold symmetry was observed, which is a consequence of periodicity that strictly forbids non-crystallographic rotational symmetries. These measurements represent a starting point for testing structural models of quasicrystals and related metallic alloys with giant unit cells by means of NMR.
\end{abstract}

Keywords: Angular-dependent spectrum; Electric field gradient; Giant unit cell; Nuclear magnetic resonance; Quasicrystals;

\title{
1 Introduction
}

Diffraction methods represent a basic tool for structural determination of quasicrystals and metallic alloys with giant unit cells [1,2]. Nevertheless, it turns out that a giant (infinite) unit cell means also a high (infinite) number of diffraction peaks, which can reach far beyond the detection capability. Consequently, not all atomic positions are unambiguously determined, which leads to structural refinement that takes into account periodicity and a model unit cell. Furthermore, different elements can have similar structural factors, which prevents to distinguish between them. Determination of atomic positions turns out to be even more difficult in case of structural disorder [3], existing both in quasicrystals as well as in metallic alloys with giant unit cells. An example is a partial site occupation resulting from occupancy smaller than 1 at certain lattice sites. Similar to that is split occupation where two neighboring sites are alternatively occupied since they cannot be occupied simultaneously. Chemical disorder results from fractional occupancy of certain lattice sites by different elements. The last example is configurational disorder resulting from statistically varying orientations of a particular subcluster inside a given cage of atoms.

Nuclear magnetic resonance (NMR) represents a complementary tool to diffraction methods. In contrast to the other spectroscopic techniques like nuclear quadrupole resonance (NQR) and Mössbauer spectroscopy it nuclear magnetic resonance (NMR) might offer effers additional information based on anisotropy of interactions between the resonant nuclei and their local environment. It turns out that the electric quadrupole interaction primarily determines the measured 
NMR spectra of quasicrystals and metallic alloys with giant unit cells. Moreover, the resonance frequency not only depends on the eigenvalues of the electric field gradient (EFG) tensor at the nucleus site, but also on the orientation of the external magnetic field with respect to the principal-axes system of the EFG tensor. The details of NMR lineshape of a spin $I=5 / 2$ are given elsewhere $[4,5,6]$. Here we remind that the NMR spectrum can be calculated by knowing the distribution of the EFG parameters $g\left(v_{Q}, \eta, \theta, \varphi\right)$, where the angles $\theta$ and $\varphi$ define the orientation of the external magnetic field $B_{0}$ with respect to the EFG tensor principal-axes system, $v_{Q}$ is the quadrupole coupling constant and is proportional to the largest eigenvalue of the EFG tensor $V_{Z Z}$, whereas $\eta=\left(V_{X X}-V_{Y Y}\right) / V_{Z Z}$ is the asymmetry parameter of the EFG tensor.

In traditional NMR, relatively large samples are needed typically of a volume of at least few cubic millimeters. In era of polygrain and powder quasicrystals, the angular-dependent information in the distribution $g\left(v_{Q}, \eta, \theta, \varphi\right)$ was lost due to averaging over all possible orientations of small crystallites. The NMR investigations confirmed an infinite number of atomic environments in quasicrystals $[7,8]$ resulting in broad NMR spectra and broad continuous distribution of EFG tensor parameters $g\left(v_{Q}, \eta, \theta, \varphi\right)$. Similar conclusions were reached also by NQR [9] and Mössbauer spectroscopy [10]. Introduction of "big" monocrystals, thanks to significant progress in metallurgy [11], offered a unique opportunity to take advantage of NMR potential for structural determination. In subsequent chapters we show that angular dependence of selectively excited NMR spectra reveals the structural symmetries of quasicrystals and metallic alloys including the observation of admired "forbidden" symmetries. This opens the possibility of dealing with unsolved problems referring to structural disorder by comparing different structural models to NMR measurements rich in structural information that are complementary to diffraction data.

\section{Decagonal AlNiCo quasicrystal}

The angular dependent NMR measurements were performed on a decagonal $\mathrm{Al}_{72.6} \mathrm{Ni}_{10.5} \mathrm{Co}_{16.9}$ singlegrain sample grown by the flux technique. The sample was rotated about the 10-fold axis as schematically shown in figure 1a. The axis of rotation was perpendicular to the external magnetic field $B_{0}$, so that the direction of the external magnetic field was swept through the $2 \mathrm{D}$ quasiperiodic layer of the decagonal quasicrystal. A typical frequency-swept ${ }^{27} \mathrm{Al}$ NMR spectrum is displayed in figure $2 \mathrm{a}$. It consists of a broad satellite "background" intensity and narrow central line [5,6]. Apart from the ${ }^{27} \mathrm{Al}$ spectrum also a part of ${ }^{59} \mathrm{Co}$ spectrum is visible. The angular dependence of the ${ }^{27} \mathrm{Al}$ spectrum is shown in figure $2 b$, pointing out that it is reasonable to measure angular dependence at the satellite part of ${ }^{27} \mathrm{Al}$ NMR spectrum. Namely, satellite transitions are predominantly determined by first-order electric quadrupole interaction, whereas the central transition is affected by interactions, such as magnetic hyperfine and RKKY interactions, in addition to the second-order electric quadrupole interaction [5].

[Insert figure 1 about here.]

Measurement of a whole ${ }^{27} \mathrm{Al} \mathrm{NMR}$ frequency-swept spectrum is time consuming as it takes approximately 48 hours. Fortunately, all needed structural information can be obtained by measuring 
the angular dependence of a selectively excited spectral intensity in a frequency window around the selected resonance frequency (see figure $2 \mathrm{~b}$ ). By doing so, we substantially improved sensitivity and angular resolution. The resulting angular dependence for rotation about the 10-fold axis of the decagonal AlNiCo quasicrystal is shown in figure 2c. A perfect 10-fold symmetry is observed with remarkable details that reflect the angular distribution of the largest eigenvalue of the EFG tensors with respect to the crystalline axes-system. Furthermore, this angular distribution is in agreement with the modeled angular distribution introduced by us (see figure $3 \mathrm{~d}$ in [6]), which was derived for periodic stacking of 2D Penrose tiling using point-charge model for calculation of EFG tensors. Compared to previous angular dependent studies of the ${ }^{27} \mathrm{Al}$ central lineshape [5], a method of measuring angular dependence of selectively excited spectral intensity at the satellite part of NMR spectrum, clearly shows its advantages in obtaining more structural information.

[Insert figure 2 about here.]

\section{Icosahedral AlPdMn quasicrystal}

Similar experiment was performed on an icosahedral $\mathrm{Al}_{70.5} \mathrm{Pd}_{21.2} \mathrm{Mn}_{8.3}$ quasicrystal grown by the Czochralski technique. The sample was rotated about one of its 5 -fold axis as shown in figure $1 \mathrm{~b}$. A rotation pattern is displayed in figure $2 \mathrm{~d}$ and again exhibits a perfect 10 -fold symmetry. Although the real space axis is 5-fold, the observed pattern has 10-fold symmetry. The electric quadrupole interaction is insensitive to $\mathbf{B}_{\mathbf{0}} \rightarrow-\mathbf{B}_{\mathbf{0}}$ transformation of external magnetic field [4], because of its tensor nature, which leads to additional 2-fold symmetry.

\section{Orthorhombic $\xi$-AlPdMn}

In the AlPdMn ternary phase diagram there exists an orthorhombic (space group Pnma) $\xi^{\prime}$-AlPdMn phase $[12,13,14]$ with giant unit cell structure. It has approximately 258 atoms per unit cell with cell parameters $a=2.354 \mathrm{~nm}, b=1.657 \mathrm{~nm}$ and $c=1.234 \mathrm{~nm}$. The skeleton of its structure is made up of pseudo-Mackay icosahedra centered on Mn atoms. These central Mn atoms are located at the vertices of flattened hexagons (figure 3a). The planes of alternatively arranged flattened hexagons are oriented perpendicular to the $\left[\begin{array}{lll}0 & 1 & 0\end{array}\right]$ direction, which represents the "pseudo" 5-fold direction of the crystal. These planes form stacked-layer structure along the $\left[\begin{array}{lll}0 & 1 & 0\end{array}\right]$ direction (figure $\left.3 b\right)$. The pseudo-Mackay icosahedra consist of a small icosahedron of $\mathrm{Al}$ atoms, a larger icosahedron of $\mathrm{Pd}$ atoms and an icosidodecahedron of $\mathrm{Al}$ atoms (figure 3a). Few additional atoms are needed to fill the empty space between the pseudo-Mackay icosahedra. This periodic crystal is also a quasicrystalline approximant to the icosahedral AlPdMn quasicrystal (having the ideal icosahedral composition $\mathrm{Al}_{70} \mathrm{Pd}_{22} \mathrm{Mn}_{8}$ ), which possesses the same local order dominated by pseudo-Mackay icosahedral clusters.

[Insert figure 3 about here.]

A monocrystal of $\xi^{\prime}$-AlPdMn was shaped in the form of rectangular prism with the long axis along the $\left[\begin{array}{lll}0 & 1 & 0\end{array}\right]$ "pseudo" 5-fold direction. It was grown by the Bridgman technique [11]. The sample 
was rotated about its "pseudo" 5-fold direction as schematically displayed in figure 1c. The rotation pattern exhibits non-perfect 10-fold symmetry including mirror plane (figure 2e) in agreement with orthorhombic unit cell and space group Pnma. This confirms the fundamental structural property of this giant-unit-cell metallic compound, which contains distorted icosahedral clusters of atoms, but lacks perfect 5-fold symmetry, which is strictly forbidden in periodic crystals. In other words, we were able to detect clusters with pseudo-icosahedral symmetry distorted by the constraint of fulfilling the periodic boundary condition.

\section{Conclusions}

Our study is a step forward in structural determination of quasicrystals and metallic alloys with giant unit cells by means of NMR., which Here it is important to stress that NMR does not address the atomic structure directly but is eomplementary to diffraction methods by being sensitive to the local symmetry of an atomic environment around the resonant nucleus. NMR is successful in determining EFG tensors around the resonant nuclei, which in turn yield the information on the number of crystallographically inequivalent atoms in the structure and hence the number of different local chemical environments. Moreover, NMR might distinguish between different possible structural models including structural disorder when diffraction methods are not conclusive. A potential of the presented technique in extracting structural information by measuring angular dependence of the selectively excited NMR intensity was already demonstrated by Klanjšek and Dolinšek [15]. In case of icosahedral AlPdMn quasicrystal the authors were able to differentiate between qualitatively different icosahedral distributions of the EFG tensor orientations, based on the angular dependence of the NMR spectral intensity about the axis that lies in the middle between two 2-fold axes. Furthermore, preliminary study on decagonal AlNiCo quasicrystal showed the ability of studying structural disorder by comparison between different structural models and NMR data [6].

\section{Acknowledgements}

We thank Michael Feuerbacher from Jülich for provision of all investigated samples. This research was supported by the Slovenian Research Agency. P. J. also acknowledges Boehringer Ingelheim Fonds for supplementary support. 
Figure 1

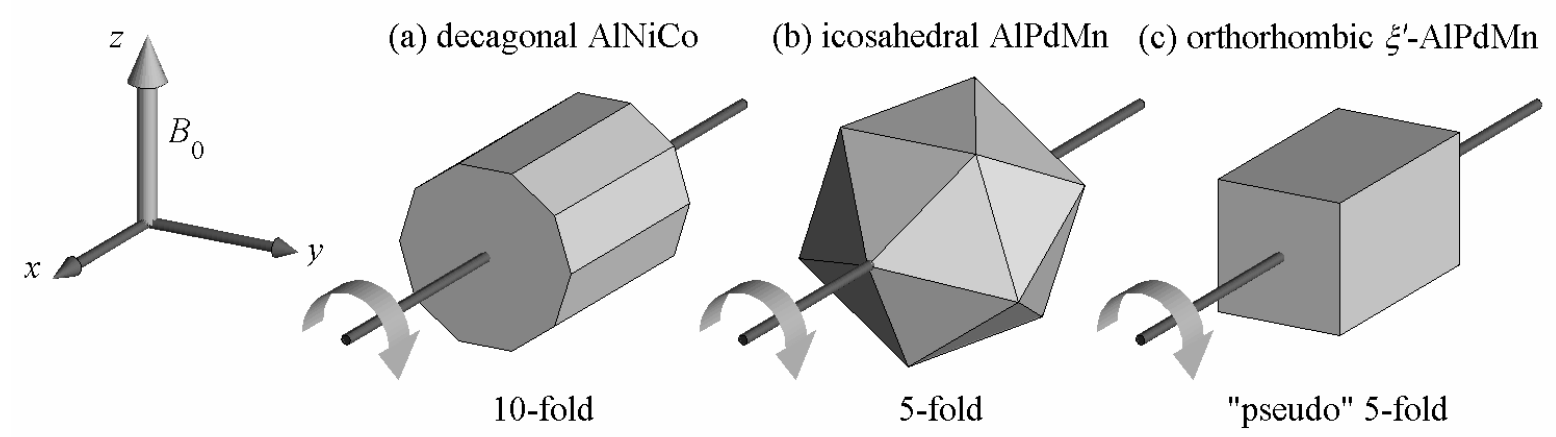


Figure 2
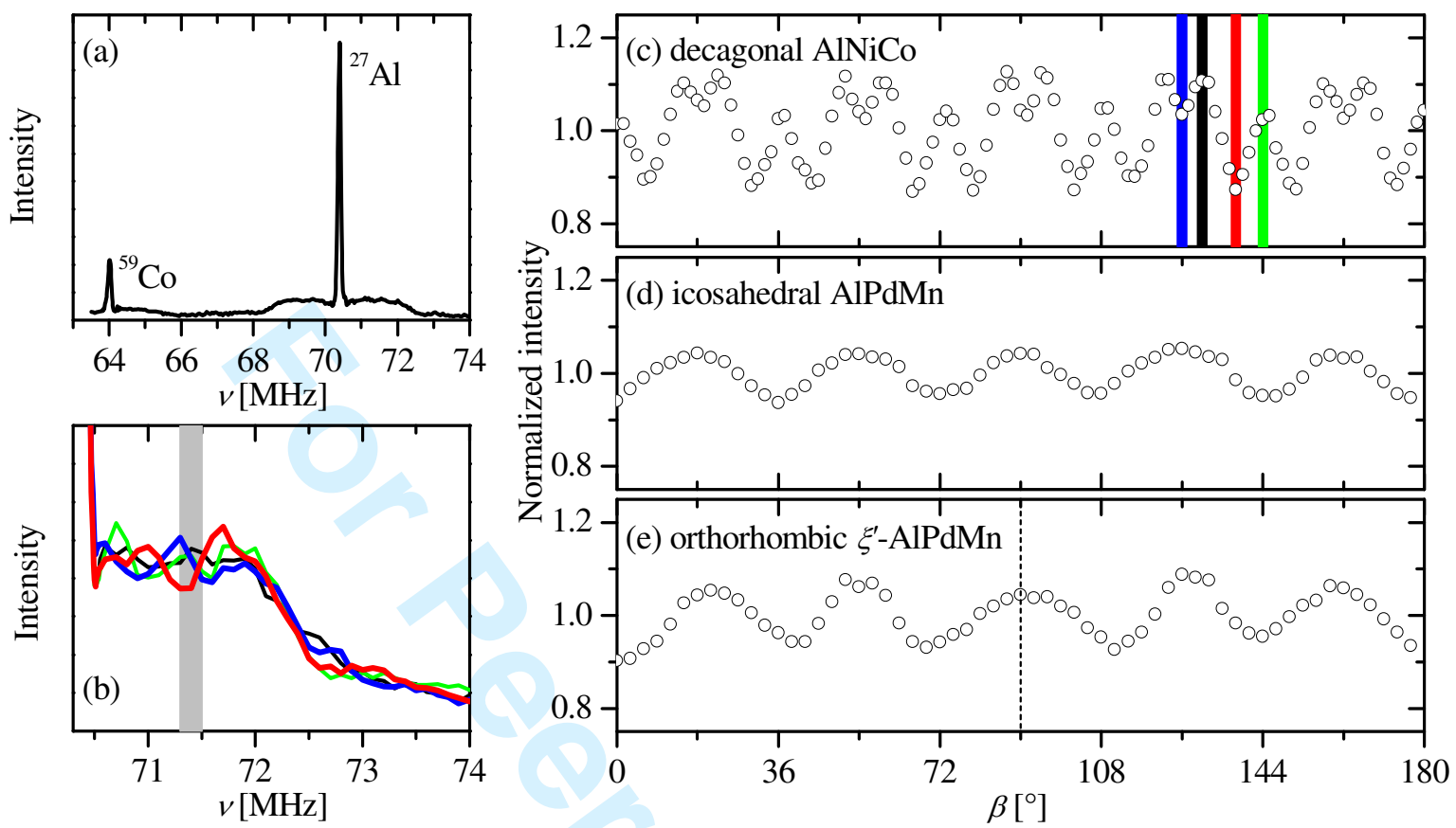
Figure 3
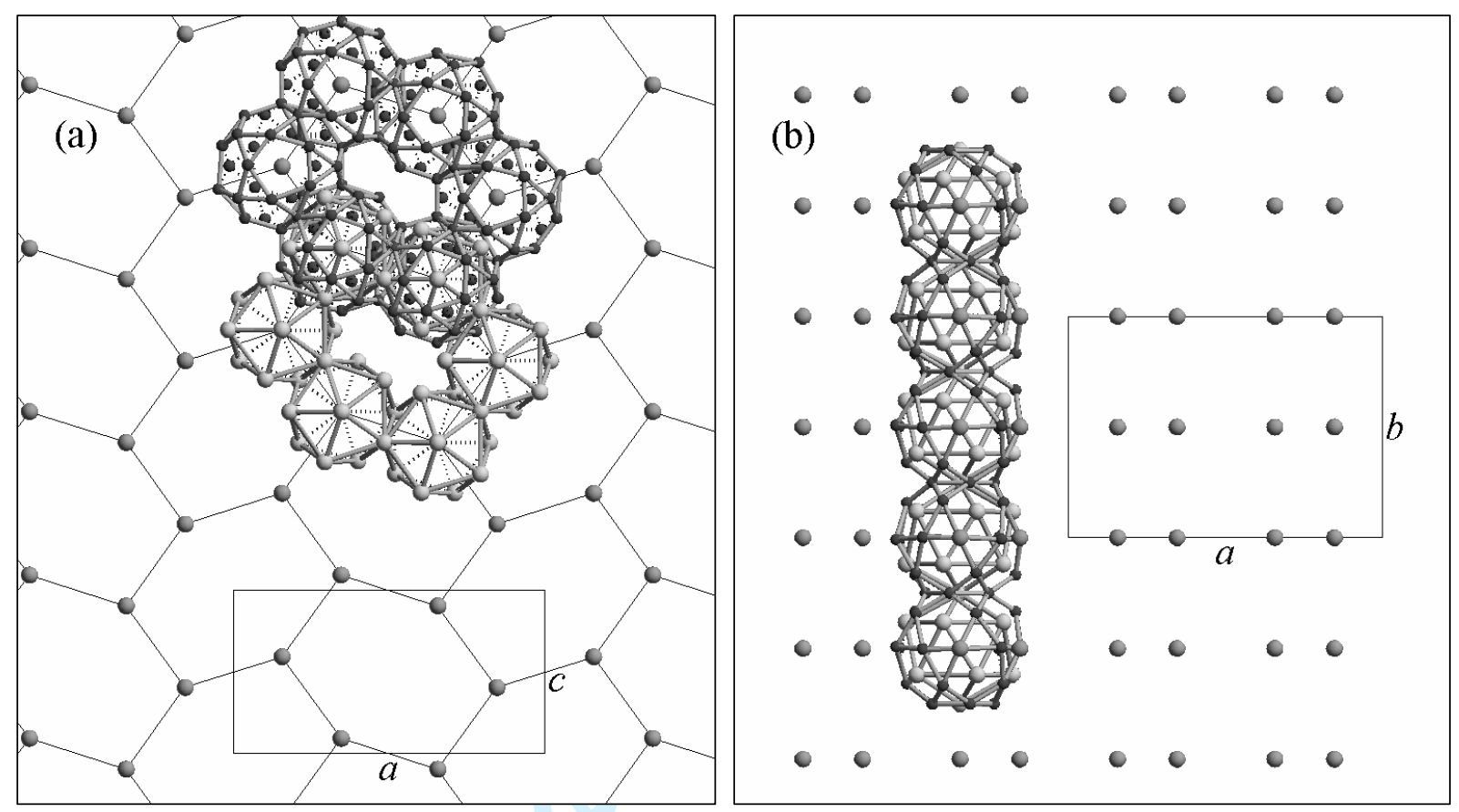


\section{Figure captions}

Figure 1. Schematic presentation of the axes of rotation used in our NMR experiments, which coincide with (a) 10-fold axis in case of decagonal AlNiCo quasicrystal, (b) 5-fold axis of icosahedral AlPdMn quasicrystal and (c) "pseudo" 5-fold axis of orthorhombic $\xi$ '-AlPdMn metallic crystal with giant unit cell. The axis of rotation is perpendicular to the direction of the external magnetic field $B_{0}$ in all cases.

Figure 2. (a) Frequency-swept NMR spectrum of a single-grain decagonal AlNiCo quasicrystal (10fold axis $\perp B_{0}, T=80 \mathrm{~K}, B_{0}=6.34 \mathrm{~T}$ ). In addition to ${ }^{27} \mathrm{Al}$ spectrum also a part of ${ }^{59} \mathrm{Co}$ spectrum is visible; (b) Selected part of ${ }^{27} \mathrm{Al}$ NMR spectra of the decagonal AlNiCo quasicrystal taken at various orientations of the sample rotated about the 10-fold axis. The shaded area indicates the region (a frequency window of $100 \mathrm{kHz}$ width around $71.4 \mathrm{MHz}$ ), where the angular dependence of selectively excited spectral intensity was measured. The latter is shown in (c), where $\beta$ is the angle describing the rotation about the 10-fold axis. The indicated positions of the angle $\beta$ coincide with colored spectra in (b). The same kind of the angular dependence is shown for (d) a single-grain icosahedral AlPdMn quasicrystal (rotation about the 5-fold axis) and (e) an orthorhombic $\xi^{\prime}$-AlPdMn monocrystal (rotation about its $\left[\begin{array}{lll}0 & 1 & 0\end{array}\right]$ direction). In the latter case non-perfect symmetry is clearly visible (mirror plane is indicated by vertical dashed line).

Figure 3. (a) Projection along the $\left[\begin{array}{lll}0 & 1 & 0\end{array}\right]$ direction representing one layer of flattened hexagons of $\mathrm{Mn}$ atoms, which is a basic structural motif of $\xi$-AlPdMn. To show the geometry and interpenetration of the outer shell of pseudo-Mackay clusters, 6 icosahedra of Pd atoms and 6 icosidodecahedra of $\mathrm{Al}$ atoms are visible. (b) Projection along the $\left[\begin{array}{lll}0 & 0 & 1\end{array}\right]$ direction showing periodic stacking of Mn layers along the "pseudo" 5-fold direction. In both cases a projection of a unit cell is shown. The cell parameters are given in text. 


\section{References}

[1] M. Boudard and M. de Boissieu, in Physical Properties of Quasicrystals, edited by Z. Stadnik (Springer-Verlag, Berlin, 1999).

[2] M. Mihalkovič, in Quasicrystals: an Introduction to Structure, Physical Properties, and Applications, edited by J.-B. Suck, M. Schreiber, P. Häusler (Springer-Verlag, Berlin, 2002).

[3] K. Urban and M. Feuerbacher, J. Non-crystalline Solids 334-335 143 (2004).

[4] J. Winter, Magnetic Resonance in Metals (Oxford University Press, Oxford, 1971).

[5] P. Jeglič and J. Dolinšek, Phys. Rev. B 71014204 (2005).

[6] P. Jeglič and J. Dolinšek, Phil. Mag. 86601 (2006).

[7] W.W. Warren, H.S. Chen, and J.J. Hause, Phys. Rev. B 327614 (1986).

[8] A. Shastri, F. Borsa, D.R. Torgeson, J.E. Shield, and A.I. Goldman, Phys. Rev. B 5015651 (1994).

[9] A. Shastri, F. Borsa, D.R. Torgeson, and A.I. Goldman, Phys. Rev. B 504224 (1994).

[10] Z.M. Stadnik, T. Takeuchi, N. Tanaka, and U. Mizutani, J. Phys. Condensed Matter 156365 (2003)

[11] M. Feuerbacher, C. Thomas, and K. Urban, in Quasicrystals: Structure and Physical Properties, edited by H.-R. Trebin (Wiley-VCH, Weinheim, 2003).

[12] H. Klein, M. Audier, M. Boudard, M. de Boissieu, L. Beraha, and M. Duneau, Phil. Mag. A 73 309 (1996).

[13] M. Boudard, H. Klein, M. de Boissieu, and M. Audier, Phil. Mag. A 74939 (1996).

[14] L. Beraha, M. Duneau, H. Klein, and M. Audier, Phil. Mag. A 76587 (1997).

[15] M. Klanjšek and J. Dolinšek, Phil. Mag. 86413 (2006). 